\title{
OBESIDADE: UMA ABORDAGEM DA PERSPECTIVA DA CULTURA CORPORAL EM ESCOLAS
}

\author{
OBESITY: AN APPROACH TO THE PERSPECTIVE OF BODY CULTURE IN \\ SCHOOLS
}

Rafael Gemin Vidal ${ }^{1}$

Bruno Cesar de Oliveira Ostrufka²

\section{RESUMO}

O presente estudo tem como objetivo fazer um levantamento bibliográfico sobre as diretrizes que regem as aulas de Educação Física escolar, fazendo apontamentos específicos sobre os parâmetros relacionados a cultura corporal, cuidados com a saúde e risco de obesidade. Sabendo que os índices de obesidade têm aumentado no Brasil, apontando os fatores biológicos e culturais como as principais causas, nota-se a importância de estruturar os conceitos da cultura corporal desde a infância, sendo papel da Educação Física escolar introduzir hábitos que possam auxiliar o estado no controle do crescimento de doenças relacionadas ao excesso de peso corporal, aumentando desta maneira a expectativa e qualidade de vida da população. Conclui-se que os parâmetros que regem as abordagens nas aulas de educação física tratam de maneira superficial a cultura corporal no ambiente escolar. Palavras-chave: Obesidade. Cultura Corporal. Educação Física escolar.

\section{ABSTRACT}

The present study aims to make a bibliographical survey about the guidelines that conduct the classes of Physical Education at school, making specific notes on the parameters related to body culture, health care and risk of obesity. Knowing that obesity rates have increased in Brazil, pointing out the biological and cultural factors as the main causes, it is important to structure the concepts of body culture from childhood, and it is the role of scholar Physical Education to introduce habits that can help the state in controlling the growth of diseases related to excess body weight, thus increasing the population's expectation and quality of life. It is concluded that the parameters that govern the approaches in physical education classes treat superficially the corporal culture in the school environment.

Keywords: Obesity. Body Culture. Scholar physical education.

Data de submissão: 06 out. 2019.

Data de aprovação: 15 maio 2020.

\footnotetext{
${ }^{1}$ Mestre em Desenvolvimento, Sociedade e Educação pela Universidade Alto Vale do Rio do Peixe (UNIARP). Professor da Unidade de Ensino Superior Vale do Iguaçu (UNIGUAÇU). Lattes: http://lattes.cnpq.br/6058590769924611. Orcid: https://orcid.org/0000-0002-5173-1095. E-mail: rafaelgemin@hotmail.com.

2 Bacharel em Educação Física pela Unidade de Ensino Superior Vale do Iguaçu (UNIGUAÇU). Lattes: http://lattes.cnpq.br/0982281069109759. Orcid: https://orcid.org/0000-0001-6895-3228. E-mail: rafaelgemin@hotmail.com.
} 


\section{INTRODUÇÃO}

A obesidade pode ser definida como uma doença crônica causada pelo excesso de peso corporal, sendo o padrão adotado pela Organização Mundial da Saúde (OMS) o índice de massa corporal para classificar os indivíduos em magreza, normal, sobrepeso, obesidade e obesidade grave. Aponta-se a obesidade como preditora de inúmeras doenças crônicas não transmissíveis (DCNTs), como diabetes tipo II, hipertensão, doenças respiratórias, metabólicas, entre outras, que resultam em uma redução da qualidade de vida (DE LACERDA et al., 2014).

Os fatores causais da obesidade são multifatoriais, sendo apontados os fatores genéticos, ecológicos, políticos, socioeconômicos, psicossociais, biológicos e culturais. Porém, os fatores mais estudados da obesidade são os biológicos relacionados ao estilo de vida, especialmente no que diz respeito ao binômio dieta e atividade física. Tais investigações se concentram nas questões relacionadas ao maior aporte energético da dieta e na redução da prática da atividade física com a incorporação do sedentarismo, configurando o denominado estilo de vida ocidental contemporâneo (WANDERLEY; FERREIRA, 2010).

O avanço tecnológico fez com que houvesse mudanças na rotina das novas gerações, onde o uso de equipamentos eletrônicos toma maior parte do dia, ora trazendo facilidades, ora apresentando certo comodismo, o que tem ocasionado um aumento significativo nos índices de sedentarismo, sobretudo em crianças e adolescentes (DE LACERDA et al., 2014).

O aumento na prevalência da obesidade na infância é preocupante devido ao risco maior dessas crianças tornarem-se adultos obesos, apresentando variadas condições mórbidas associadas. A obesidade e o sedentarismo são importantes fatores de risco para o desenvolvimento de doenças cardiovasculares, sendo a prática de atividade física fundamental como forma de prevenção (GUEDES; GUEDES, 2003).

Analisando que hábitos que permanecem no decorrer da vida são adquiridos na infância, alguns fatores negativos, se identificados precocemente, possuem maiores chances de serem modificados, como o comportamento sedentário, que apresenta grande incidência nos adolescentes da atualidade, pois o pouco interesse aos hábitos que necessitam de maior 
demanda energética é inversamente proporcional ao interesse da comodidade tecnológica de celulares e computadores.

A escola tem papel de formação do ser humano, através de um processo onde a criança se converte num adulto que compreende o agrupamento de crenças e hábitos da sociedade, sendo que a Educação Física, além das vivências esportivas, tem função de estimular o conhecimento corporal, implementando valores de hábitos saudáveis, contribuindo, desta maneira, para formação de uma geração com condutas adequadas no que se refere a saúde, corpo e qualidade de vida.

Sabendo que os índices de obesidade têm aumentado no Brasil, apontando os fatores biológicos e culturais como as principais causas, nota-se a importância de estruturar os conceitos da cultura corporal desde a infância, sendo papel da Educação Física escolar introduzir hábitos que possam auxiliar o estado no controle do crescimento de doenças relacionadas ao excesso de peso corporal, aumentando, assim, a expectativa e qualidade de vida da população.

Portanto, o presente estudo tem como objetivo fazer um levantamento bibliográfico sobre as diretrizes que regem as aulas de Educação Física escolar, fazendo apontamentos específicos sobre os parâmetros relacionados a cultura corporal, cuidados com a saúde e risco de obesidade.

Como meio metodológico adotou-se de uma pesquisa básica, qualitativa e descritiva bibliográfica, que realizou consulta em artigos publicados nas bases de dados Google Acadêmico, Scielo, Scopus e Lilacs, utilizando dos descritores "Cultura Corporal" and "Obesidade" and "Educação Física escolar", para os artigos publicados em português entre os anos de 2008 e 2019, além da concordância com os documentos regimentares da educação, Parâmetros Curriculares Nacionais (PCNs) e Base Nacional Comum Curricular (BNCC).

\section{OBESIDADE}

Entende-se por sobrepeso e obesidade o acúmulo anormal ou excessivo de gordura que pode apresentar riscos para a saúde. Em adultos, as variáveis de sobrepeso e obesidade são determinadas usando Índice de Massa Corporal (IMC), que se obtém dividindo o peso em 
quilogramas pelo quadrado da altura em metros $(\mathrm{kg} / \mathrm{m} 2)$. A Organização Mundial da Saúde (OMS) define um IMC igual ou superior a 25 como sobrepeso e um IMC igual ou maior que 30 como obesidade.

O sobrepeso e a obesidade são resultados de um desequilíbrio energético oriundo de uma excessiva ingestão calórica e a falta de atividade física para consumir estas calorias. Esta mudança nos padrões alimentares e de exercícios físicos resultam de padrões ambientais e socioculturais associados ao desenvolvimento constante e a falta de políticas de apoio em setores como saúde, transporte, planejamento urbano, processamento de alimentos, marketing e educação.

Os níveis de obesidade que atingem crianças e adolescentes em todo o mundo tiveram um aumento de menos de $1 \%$ (equivalente a cinco milhões de meninas e seis milhões de meninos) em 1975, saltando para quase $6 \%$ em meninas (50 milhões) e quase $8 \%$ em meninos (74 milhões) em 2016. Combinado a isso, o número de obesos com idade entre 5 e 19 anos cresceu dez vezes mais, de 11 milhões em 1975 para 124 milhões em 2016. Outros 213 milhões estavam com sobrepeso em 2016, mas o número caiu abaixo do limiar para a obesidade (LEANDRA et al., 2017).

A obesidade infantil se associa a uma maior chance de desenvolvimento de dissociações metabólicas, dificuldade respiratória, aumento do risco de fraturas, hipertensão, marcadores precoces de doença cardiovascular, resistência à insulina, efeitos psicológicos, risco de morte prematura e incapacidade funcional na idade adulta. Crianças em países pobres e em desenvolvimento são muito mais vulneráveis à quadros de desnutrição e, ao mesmo tempo, são expostos a alimentos com alto teor de gorduras, açúcar, alta quantidade de sal e alimentos pobres em micronutrientes, que, na maioria das vezes, tem um baixo custo de produção. Esses padrões de alimentação, em conjunto com baixos níveis de atividade física, resultam e colaboram para o aumento da obesidade, enquanto isso os problemas com desnutrição ainda parecem não ter uma solução. Segundo a Organização Pan-Americana de Saúde Brasil (OPAS, 2016) 41 milhões de crianças com menos de 5 anos estavam com sobrepeso ou obesas em 2014. A obesidade é uma realidade crescente nas diferentes regiões do Brasil, o que vai de encontro com vários estudos relacionando as causas e consequências (JARDIM; SOUZA, 2017). 
O sobrepeso e a obesidade, tanto quanto as DCNTs associadas, podem ser evitadas e é fundamental moldar as pessoas e guiá-las para escolhas mais saudáveis em seus estilos de vida e cultura corporal, grupos de apoio, ambientes socio-educativos, programas educacionais para os ensinos fundamentais e de base são caminhos para um futuro contra o sobrepeso e a obesidade.

\section{CULTURA CORPORAL NA ESCOLA}

Segundo Zottis (2002), pessoas com corpos obesos encontram uma dificuldade em desempenhar tarefas cotidianas fáceis que vão desde atividades domésticas até a própria higiene corporal. Essas dificuldades acabam se tornando uma motivação para diminuir o peso corporal e, assim, retomar a vida cotidiana normalmente, recuperando sua autoestima. Como a estética é supervalorizada e, consequentemente, a rejeição social sofrida pelo obeso se torna evidente, é notório o número crescente de casos de depressão emocional e vários outros distúrbios de comportamento e autoimagem, motivo pelo qual é faz-se necessário compreender a percepção que o corpo obeso tem sobre sua própria imagem.

A preocupação com a imagem corporal não é mais uma questão só dos adultos, mas hoje, principalmente, dos adolescentes, os quais estão passando por uma fase de mudanças. Sendo assim, querem, de qualquer forma, fazer parte desse modelo de corpo que a sociedade impõe atualmente (OLIVEIRA, 2013).

Nos últimos 15 anos, o crescimento em torno da cultura corporal e esportiva vem sendo um dos destaques mais importantes nos meios de comunicação de massa e na economia. As práticas de atividade física tornam-se, cada dia mais, produtos de consumo. As mídias, a cada dia, divulgam incessantemente ideias sobre a cultura corporal do movimento, sendo que, na maioria das divulgações, o alvo é o público adolescente (BETTI; ZULIANI, 2002).

O estilo de vida imposto pelas condições socioeconômicas da atualidade (consumismo, desemprego crescente, informatização e automatização do trabalho, deterioração dos espaços públicos de lazer, violência, poluição) leva a uma enorme quantidade de pessoas ao sedentarismo, à má alimentação, estresse etc. O aumento do tempo em frente à televisão, especialmente por parte das crianças e adolescentes, diminuindo a atividade física, traz 
desinteresse à cultura de jogos infantis e incentiva a substituição da vontade de praticar esporte pela de assistir esporte. Essa situação cria um questionamento da atual prática pedagógica da Educação Física escolar por parte dos alunos que, não vendo mais "graça" na disciplina, encontram ou até mesmo criam situações para a dispensa da prática. Contudo, dão mais valor as práticas corporais realizadas fora da escola. O fenômeno é mais visível no Ensino Médio, onde tem-se uma linha de pensamento advinda dos adolescentes mais independente e autônoma, comparada aos anos iniciais.

As Diretrizes Curriculares do Estado do Paraná preconizam, no ensino básico da Educação Física, uma metodologia que contribua, através da cultura do movimento, para capacitar aluno a perceber os componentes sociais que influenciam todas as ações socioculturais, se desenvolvendo mentalmente para posicionar-se responsavelmente de maneira construtiva e crítica, usando maneiras lógicas e diálogos para mediar e resolver seus conflitos e tomadas de decisão (PARANÁ, 2008).

O objetivo dos Parâmetros Curriculares Nacionais (PCNs) é a evolução da base de ensino, onde os jovens e adolescentes estejam preparados para compreender a cidadania, sendo socialmente ativos, conscientes de seus direitos e deveres políticos, civis e sociais, tendo e praticando a solidariedade, a cooperação, a aversão à injustiça e a empatia. Posteriormente, adquirir conhecimentos do cenário nacional em suas diversas dimensões, sejam elas sociais, materiais e culturais e, com isso, sua identidade nacional e patriotismo, conhecendo o valor da diversidade sociocultural brasileira e de outros povos, tornando-se analítico para repudiar qualquer intolerância cultural, social, crenças, gênero, etnia e peculiaridades individuais e sociais. Nesse contexto, entra-se com a cultura corporal, que busca o desenvolvimento aprofundado sobre si mesmo, o conhecimento em relação a autoconfiança em suas capacidades físicas, afetiva, cognitiva, ética, estética, inter-relação pessoal e social. Conhecer o próprio corpo e, assim, o preservar, sendo zeloso e adquirindo hábitos saudáveis para uma melhor qualidade de vida individual e coletiva (BRASIL, 1998).

Grande parte da população ainda possui um comportamento análogo em relação à prática de exercício físico e saúde, pois, mesmo tendo a consciência de que a prática regular de exercícios físicos promove resultados positivo para a saúde, poucas ainda aderem a esse estilo de vida, se tornando um desafio ainda maior para o educador físico, o qual tem como objetivo mudar esse quadro ainda existente (MELLO; VAISBERG, 2010). 
O combate a esse desinteresse exige que o profissional de Educação Física encontre novos métodos para a aplicabilidade da cultura corporal, usando das mais diversas vertentes que a cultura do movimento dispõe e, assim, tenha uma junção entre as diretrizes curriculares que regem a educação na escola e o modo como essa mesma educação é colocada para os adolescentes, criando um vínculo mais forte entre professor-aluno, podendo ter uma influência maior em relação à maneira como o jovem se vê e a cultura corporal imposta pelos padrões sociais da atualidade (BETTI; ZULIANI, 2002).

Uma variedade de elementos pode afetar os alunos por toda a vida, podendo-se incluir a condição dos sistemas fisiológicos, características psicológicas, solicitação de tarefas motoras, entre outros (GALLAHUE, 2013). Essa decadência é dada aos hábitos de vida que, dependendo quais forem criados, acarretam sobrepeso e obesidade, distúrbios psicológicos e fisiológicos que, muitas vezes, são esquecidos ou nem mesmo trabalhados de maneira educativa nas fases fundamentais do ensino.

Se torna importante, portanto, que ocorra o desenvolvimento de estratégias com o objetivo de aumentar a prática de exercícios físicos regulares dos escolares. Por isso, sugerese que sejam inseridas atividades que, além de tudo, sejam prazerosas e estimulem a vontade de que a criança e/ou adolescente continue a prática e a torne como um hábito incluso na sua rotina.

\section{CONSIDERAÇÕES FINAIS}

Diante das discussões geradas e tendo por objetivo analisar o ambiente da cultura corporal no ensino da educação física escolar, relacionando este fato com os crescentes dados de obesidade no país, sobretudo em crianças e adolescentes, conclui-se que os parâmetros que regem as abordagens nas aulas de educação física tratam de maneira superficial a cultura corporal no ambiente escolar.

É fato que a geração se molda constantemente, sendo que a atual já nasce estimulada por diversos meios tecnológicos, o que tem gerado, em parte, o aumento do sedentarismo e, como consequência, o aumento da massa corporal total, que levam ao sobrepeso e à obesidade. 
Estudos realizados em escolares têm mostrado a prevalência de sobrepeso e obesidade em escolares, estando estes ligados diretamente com as práticas de atividades físicas realizadas por crianças e adolescentes (BERTUOL; NAVARRO, 2015; TEIXEIRA et al., 2015; BALABAN, 2001).

A prática regular de atividades físicas é hábito, que deve ser incentivado desde a primeira infância. Portanto, a educação física escolar tem como missão difundir, através da prática de exercícios corporais, valores e condutas, que reflitam positivamente em todo o decorrer da vida.

O contexto desta temática exige atenção dos profissionais de Educação Física, assim como gestores e orientadores. Uma visão crítica dos parâmetros curriculares se faz necessária, assim como uma abordagem incisiva da cultura corporal dentro do ambiente escolar, para difusão da prática regular de exercícios físicos, combatendo direta e indiretamente a incidência de sobrepeso e obesidade e, consequentemente, das doenças crônicas não transmissíveis, além da inclusão de valores morais e éticos através de suas práticas, sendo, portanto, uma medida de ação social, cultural, relacional, de saúde e educação.

\section{REFERÊNCIAS}

ABARCA-GÓMEZ, L. et al. Worldwide trends in body-mass index, underweight, overweight, and obesity from 1975 to 2016: a pooled analysis of 2416 population-based measurement studies in 128.9 million children, adolescents, and adults. The Lancet, v. 390, i. 10113, p. 2627-2642, dec. 2017. Disponível em: https://bit.ly/3cfvnQd. Acesso em: 30 maio 2020.

BALABAN, G.; SILVA, G. A. P. Prevalência de sobrepeso e obesidade em crianças e adolescentes de uma escola da rede privada de Recife. J. Pediatr., Rio de Janeiro, v. 77, n. 2, p. 96-100, 2001. Disponível em: https://bit.ly/2zKUihp. Acesso em: 30 maio 2020.

BERTUOL, C. D.; NAVARRO, A. C. Consumo alimentar e prevalência de obesidade/emagrecimento em pré-escolares de uma escola infantil pública. RBONE Revista Brasileira de Obesidade, Nutrição e Emagrecimento, v. 9, n. 52, p. 127-134, 2015. Disponível em: https://bit.ly/2Mg17u4. Acesso em: 30 maio 2020.

BETTI, M.; ZULIANI, L. R. Educação física escolar: uma proposta de diretrizes pedagógicas. Revista Mackenzie de Educação Física e Esporte, v.1, n. 1, 2002. Disponível em: https://bit.ly/2XKnkFV. Acesso em: 30 maio 2020. 
BRASIL. Secretaria de Educação Fundamental. Parâmetros curriculares nacionais: educação física. Brasília: MEC, 1998.

DE LACERDA, L. R. F. et al. Prevalência de obesidade infantil e sobrepeso em escolares. Revista Interfaces: Saúde, Humanas e Tecnologia, v. 2, n. 5, 2014. Disponível em: https://bit.ly/2XIT6TQ. Acesso em: 30 maio 2020.

GALLAHUE, D. L. Compreendendo o desenvolvimento motor: bebês, crianças, adolescentes e adultos. 7. ed. Porto Alegre: AMGH, 2013.

GUEDES, D. P.; GUEDES, J. E. R. P. Controle do peso corporal: composição corporal atividade física e nutrição. 2. ed. Rio de Janeiro: Shape, 2003.

JARDIM, J. B.; DE SOUZA, I. L. Obesidade infantil no Brasil: uma revisão integrativa. JMPHC Journal of Management \& Primary Health Care, v. 8, n. 1, p. 66-90, 20 ago. 2017. Disponível em: https://doi.org/10.14295/imphc.v8i1.275. Acesso em: 30 maio 2020.

KOWALSKI, M.; FERREIRA, M. B. R. Estética, corpo e cultura. Conexões, v. 5, n. 2, p. 89-112, 2007. Disponível em: https://doi.org/10.20396/conex.v5i2.8637881. Acesso em: 30 maio 2020.

MELLO, M. T.; VASIBERG, M. Exercícios na saúde e na doença. São Paulo: Manole, 2010.

OLIVEIRA, N. A. A imagem corporal dos adolescentes: a busca incessante pelo corpo perfeito. EFDeportes.com, Buenos Aires, año 18, n. 180, mayo de 2013. Disponível em: https://bit.ly/2XgDnMu. Acesso em: 30 maio 2020.

PARANÁ (Estado). Secretaria de Estado da Educação. Diretrizes Curriculares Orientadoras da Educação Básica para a Rede Pública Estadual do Paraná. Curitiba: SEED, 2008.

SALVE, M. G. C. et al. Obesidade e peso corporal: riscos e consequências. Movimento \& Percepção, v. 6, n. 8, p. 29-48, 2006. Disponível em: https://bit.ly/2TWg7Bo. Acesso em: 30 maio 2020.

TEIXEIRA, A. L. S.; DESTRO, D. S. Obesidade infantil e educação física escolar: possibilidades pedagógicas. Revista Eletrônica da Faculdade Metodista Granbery, v. 9, p. 1-15, 2010.

TEIXEIRA, C. S. et al. Transtornos alimentares em adolescentes de uma escola estadual do noroeste paulista. Arquivos de Ciências da Saúde, v. 22, n. 2, p. 84-87, jul. 2015. Disponível em: https://bit.ly/2ZRw3J5. Acesso em: 30 maio 2020.

WANDERLEY, E. N.; FERREIRA, V. A. Obesidade: uma perspectiva plural. Ciência \& Saúde Coletiva, v. 15, n. 1, p. 185-194, 2010. Disponível em: https://bit.ly/2XhwKcX. Acesso em: 30 maio 2020. 
ZOTTIS, C.; SOUZA, R.H.S.; BURIN, D.A P. Gastroplastia: uma alternativa para a qualidade de vida no tratamento da obesidade mórbida. In: CONGRESSO BRASILEIRO DE ENFERMAGEM, 54., 2002, Fortaleza. Anais [...]. Fortaleza: ABEn, 2002. 